\title{
Characterization of Norovirus and Other Human Enteric Viruses in Sewage and Stool Samples Through Next-Generation Sequencing
}

\author{
Sofia Strubbia ${ }^{1}$ My V. T. Phan ${ }^{2}$ Julien Schaeffer ${ }^{1} \cdot$ Marion Koopmans $^{2} \cdot$ Matthew Cotten ${ }^{2,3,4,5}$. \\ Françoise S. Le Guyader ${ }^{1}$ (D)
}

Received: 5 July 2019 / Accepted: 17 August 2019 / Published online: 24 August 2019

(c) The Author(s) 2019

\begin{abstract}
This study aimed to optimize a method to identify human enteric viruses in sewage and stool samples using random primed next-generation sequencing. We tested three methods, two employed virus enrichment based on the binding properties of the viral capsid using pig-mucin capture or by selecting viral RNA prior to library preparation through a capture using the SureSelect target enrichment. The third method was based on a non-specific biophysical precipitation with polyethylene glycol. Full genomes of a number of common human enteric viruses including norovirus, rotavirus, husavirus, enterovirus and astrovirus were obtained. In stool samples full norovirus genome were detected as well as partial enterovirus genome. A variety of norovirus sequences was detected in sewage samples, with genogroup II being more prevalent. Interestingly, the pig-mucin capture enhanced not only the recovery of norovirus and rotavirus but also recovery of astrovirus, sapovirus and husavirus. Documenting sewage virome using these methods provides information for molecular epidemiology and may be useful in developing strategies to prevent further spread of viruses.
\end{abstract}

Keywords Human enteric viruses $\cdot$ Norovirus $\cdot$ Sewage $\cdot$ Metagenomic $\cdot$ Virome

\section{Introduction}

Increasing human population leads to increased demand for agricultural products and water, wastewater re-use will be necessary, which will pose a risk for virus pollution of the environment and subsequent affects viral transmission (Sano et al. 2016). More than 100 species of enteric viruses have been identified in human feces and in sewage such as astroviruses, caliciviruses, enteroviruses, enteric adenoviruses, and rotaviruses (Fernandez-Cassi et al. 2018; Hoque et al. 2019;

Françoise S. Le Guyader

sleguyad@ifremer.fr

1 Ifremer, Laboratoire de Microbiologie, LSEM-SG2M, BP 21105, 44311 Nantes Cedex 3, France

2 Department of Viroscience, Erasmus MC, University Medical Center Rotterdam, Rotterdam, The Netherlands

3 Present Address: London School of Hygiene and Tropical Medicine, London, UK

4 Present Address: Uganda Virus Research Institute, Entebbe, Uganda

5 Present Address: MRC-Centre for Virus Research, Glasgow, UK
Metcalf et al. 1995; Gerba et al. 2018). Documenting viral prevalence and diversity in sewage may be a useful method for monitoring viruses circulating in the community (Smith et al. 2016). However, their detection by PCR approaches requires previously identified sequences for primer design and will not detect more distant viruses.

Metagenomics refer to the description of all nucleic acids sequences present in a sample (Forbes et al. 2017). Most of human enteric viruses have small RNA genomes making their detection difficult. Indeed, their relative abundance if compared to bacteria, phage, parasites present in the environment challenge their detection and identification (Cotten and Koopmans 2016; Nieuwenhuijse and Koopmans, 2017; Adriaenssens et al. 2018). Thus, viral metagenomics is generally performed by removing as much host and bacteria as possible followed by nuclease treatment to remove free nucleic acids (Kim et al. 2017; Nieuwenhuijse et al. 2017). Detection of viruses can be performed after de novo assembly of short-read data into longer sequences (contigs) followed by a variety of computational methods for detecting known and novel viral sequences (Cotten et al. 2014; Cotten et al. 2016; Oakeson et al. 2017). However, each sample type is unique and methods need to be adapted to account for 
origin and to address the metagenomic objective. A concrete example was provided by the analysis of samples collected during the Tara oceans expedition (Alberti et al. 2017). The strategy applied allowed generation of data from a variety of organisms, including viruses and plankton from oceanic samples collected worldwide. Their approach showed the importance of using separate processing steps to analyze the different compartments of a volume of (sea)water. To identify human enteric viruses in complex samples such as stool or sewage samples, a method able to select these small particles resistant to acidic conditions, to eliminate bacteria and to decrease as much as possible background genomes such as phage genomes will be useful.

The aim of the current study was to evaluate methods for human enteric virus detection using metagenomics with a focus on norovirus. Noroviruses have great genomic diversity and they are divided into seven genogroups and many genotypes based upon genomic sequence phylogeny (de Graaf et al. 2017). Three of these genogroups (GI, GII and GIV) infect humans, and constitute the principal agent of acute gastroenteritis worldwide. Importantly for environmental research norovirus are excreted at high concentrations by infected individuals and they are highly persistent (Atmar et al. 2018). Their concentrations in sewage, that may vary among countries, are usually high and their presence in waters constitutes a major public health issue nowadays (Sano et al. 2016; Schaeffer et al. 2018). To specifically enrich our metagenomics libraries in norovirus sequences, we selected three protocols that take advantage of known properties of these viruses. The first method uses SureSelect target enrichment (Agilent) with probes matching human norovirus sequences. The second method comprises $\mathrm{pH}$ variations, based on norovirus capsids isoelectric point and on their resistance to both high and low $\mathrm{pH}$. The third method is based on norovirus binding to glycans structures that are present in human but also pig mucins.

\section{Materials and Methods}

\section{Samples}

Five human stool samples (sample 570, 287, 5, 581, 582) positive for norovirus collected between January 2008 and October 2016 were used as a $10 \%$ suspension in water. Four samples were analyzed following method $B$ and one sample following method B and C (stool sample 570) (Fig. 1). Seven raw sewage samples were collected from a gathering point upstream of any process between January 2014 and February 2017 from different sewage treatment plants located in the southern part of Brittany (France). Three sewage samples (sample 1777, 1797, 1854) were analyzed using method A

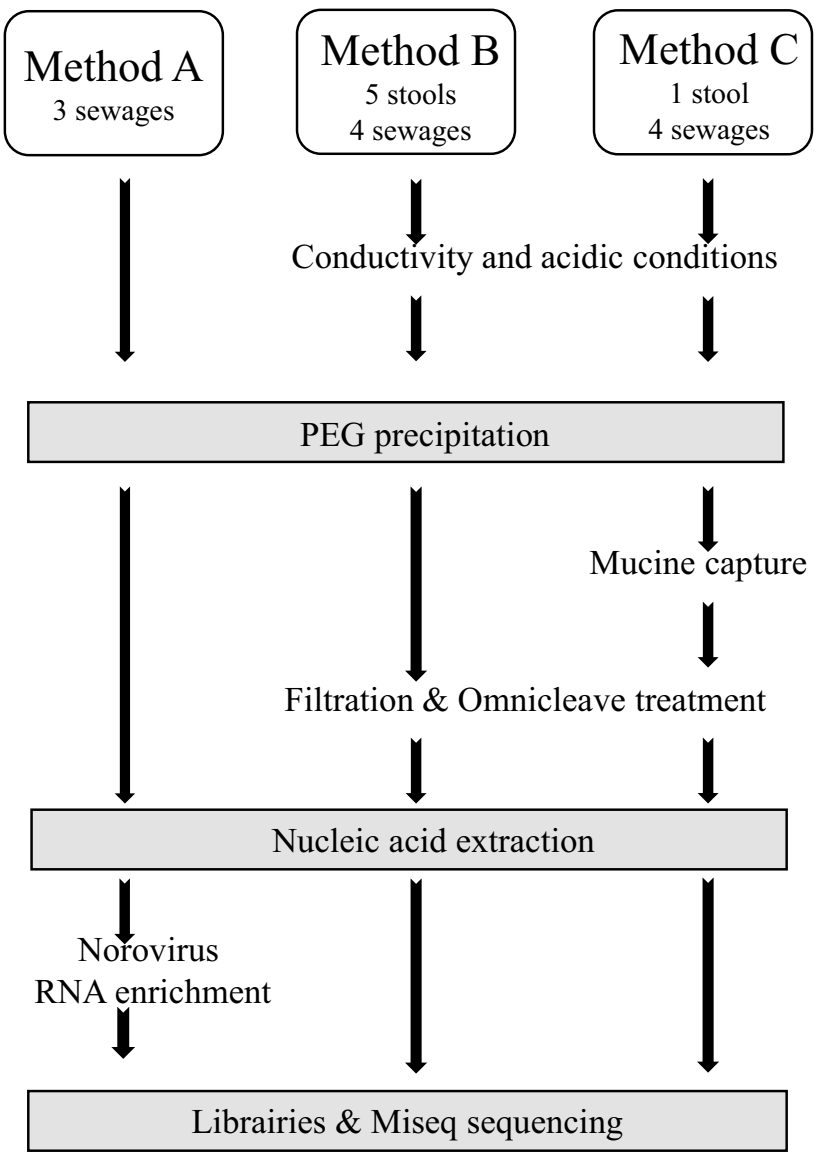

Fig. 1 Schematic of sample processing and sequencing used in this study. The three main frames on the top contain the method names (A, B and C), the number and the type of sample treated with each protocol. Key steps common to all protocols are represented in gray rectangle

and the remaining four samples (sample 1887, 1919, 1920, 1927) were analyzed using method B and C.

\section{Method A: Polyethylene Glycol Precipitation and Norovirus SureSelect Target}

Sewage samples were concentrated using a polyethylene glycol precipitation method (PEG) as previously described (Lewis et al. 1988; Schaeffer et al. 2018). Briefly, $40 \mathrm{~mL}$ of sample were mixed with $10 \mathrm{~mL}$ of a 50\% PEG 6000 solution (Sigma-Aldrich, St Quentin France) (Fig. 1). After gentle agitation overnight at $4{ }^{\circ} \mathrm{C}$, the mixture was centrifuged for $1.5 \mathrm{~h}$ at $13,500 \times \mathrm{g}$ and the pellet was suspended in $3 \mathrm{~mL}$ of $0.05 \mathrm{M}$ glycine buffer ( $\mathrm{pH} 9$ ). After nucleic acid extraction as described below, norovirus RNA sequence were enriched using the SureSelect target enrichment specific array during the library preparation (details in the library preparation paragraph) (Brown et al., 2016). 


\section{Method B: Acidic Treatment and PEG Precipitation}

To optimize human enteric viruses recovery, viruses were acid adsorbed to organic or inorganic particles present in the samples by increasing the conductivity of stool suspension or sewage samples to $2000 \mu \mathrm{S}$ by addition of $5 \mathrm{M}$ sodium chloride at pH 3 (Mullendore et al. 2001), and then concentrated using PEG precipitation as described above (Fig. 1). After centrifugation, the pellet was suspended in $3 \mathrm{~mL}$ of $0.05 \mathrm{M}$ glycine buffer ( $\mathrm{pH} 9$ ), and filtered through 5, 1.2, 0.45 and $0.22 \mu \mathrm{M}$ filters (Minisart NML 17594, NML17593, PES16533, PES16532). Then, the filtrate was incubated with 2000 Units of OmniCleave Endonuclease (Epicentre, Madison, USA) for one hour at $37^{\circ} \mathrm{C}$ to eliminate free nucleic acids, followed by nucleic acid extraction.

\section{Method C: Porcine Gastric Mucin (PGM) Capture}

Samples were prepared following method B and after incubation with the OmniCleave Endonuclease, porcine gastric mucin (PGM) capture was applied (Fig. 1). Type III PGM ( $7.5 \mathrm{mg} / \mathrm{mL}$, Sigma, France) was conjugated to MagnaBind TM carboxyl-derivatized beads (Pierce Biotechnology, Rockford, IL, USA) according to the manufacturer's protocol. Subsequently, $100 \mu \mathrm{L}$ of PGM-bead suspension was mixed for 1 hour at room temperature with $3 \mathrm{~mL}$ glycine buffer PEG sewage concentrate (Tian et al. 2008). After capturing the beads using a magnetic rack, the supernatant was discarded and the beads were released and suspended in $1 \mathrm{~mL}$ of sterile water. This volume was further diluted in the lysis buffer for nucleic acid extraction as described below.

\section{Nucleic Acid Extraction}

Nucleic acids were extracted from sample concentrates prepared using the three methods by adding $10 \mathrm{~mL}$ of the chaotropic agent guanidine thiocyanate reagent lysis buffer (bioMerieux, Lyon, France) and incubation for $10 \mathrm{~min}$ at room temperature. Then, for samples prepared using Method C, the supernatants were transferred to new tubes after bead capture using the magnetic rack. Then, $140 \mu \mathrm{L}$ of paramagnetic silica bead suspension was added (NucliSens kit, bioMerieux) to all the tubes and further incubated for $10 \mathrm{~min}$ at room temperature. The beads were captured using the magnetic rack and the volume was reduced to $2 \mathrm{~mL}$ for further extraction and purification steps as recommended (Schaeffer et al. 2018). A final step of RNA cleaning and concentration step was performed using a Zymo-spin column (RNA Clean \& Concentrator, Zymo Research, Irvine, USA). The final step was a DNAse treatment for $30 \mathrm{~min}$ at $37{ }^{\circ} \mathrm{C}$ with 5 Units of Turbo DNAse, (Ambion, ThermoFisher Scientific, France) (method B and C).

\section{Norovirus Quantification}

A microfluidic-based digital one-step RT-PCR, that allow quantification without external calibration curves was performed using primers and probes targeting the ORF1-2 region (Polo et al. 2016). Positive and negative controls were included in each series, and quantification was calculated using the Poisson distribution (QuantStudio ${ }^{\mathrm{TM}}$ 3D Analysis Suite ${ }^{\mathrm{TM}}$ Cloud Software, version 3.0.3; ThermoFisher). The final result was expressed as RNA copies/ $\mu$.

\section{Library and Sequencing}

For method A, cDNA were synthesized using Superscripts II and random primers according to the manufacturer protocol (Life Technologies). The second-strand cDNA synthesis was performed using $5 \mathrm{U}$ of Klenow (Invitrogen) polymerase in a final volume of $30 \mu \mathrm{L}$ followed by SureSelect enrichment (Agilent) with the RNA bait design previously designed (Brown et al. 2016). NGS librairies were prepared using a SureSelect ${ }^{\mathrm{XT}}$ Illumina paired-end sequencing library protocol (Agilent).

For methods B and C, after cDNA synthesis using SuperScript II and random primers according to the manufacturer's protocol, the second-strand DNA was synthesized according to the manufacturer's protocol (New England BioLabs). NGS libraries were prepared using the NEB Next Ultra DNA Library Prep Kit for Illumina (New England BioLabs) according to the manufacturer's instructions.

Sequencing was performed on the Illumina MiSeq platform, with Phage PhiX174 added to samples to standardize the runs.

\section{Sequence Analysis}

Illumina adapters were removed from the raw reads and resulting reads were trimmed using QUASR (Watson et al. 2013) from the $3^{\prime}$ end to reach a median Phred score $\geq 35$, which means a base call accuracy between $99.9 \%$ and $99.99 \%$. Reads shorter than $80 \%$ of the original read length were discarded.

\section{General De Novo Assembly}

De novo assembly was performed with quality-controlled reads using SPAdes v.3.10.1 (Bankevich et al. 2012). A variety of assembly conditions were examined but in general assembly with no error correction or read normalization yielded the largest initial contig set. Virus specific contigs were identified using Usearch (Edgar 2010), against a set of 
39 virus family or subfamily specific protein databases and virus family specific contigs were further assembled into larger contigs using mapping of the contigs against the closest identified full genome. A penultimate consensus genome was generated from the contigs. A final check of the genome was performed by mapping all quality-controlled reads to the penultimate consensus genome and a final majority nucleotide consensus genome was generated. All expected reading frames were examined, any disruption was checked and resolved by consulting the original reads across the query site.

\section{Virus Family Specific De Novo Assembly}

All virus family-specific reads were then harvested by mapping to a comprehensive set of all sequences $>500 \mathrm{nt}$ available for that family in GenBank using Bowtie2 (Langmead et al. 2012). The resulting virus family-specific reads were de novo assembled using SPAdes v.3.10.1 (Bankevich et al. 2012). Contigs shorter than 500nt were removed from subsequent analysis and contigs with coverage below 10 (determined by Bowtie 2 mapping) were carefully examined to avoid assembling contigs with varying coverage. Further assembly and genome checking were performed as described above.

\section{Results}

One objective of this work was to explore three biochemical and nucleic acid enrichment methods in sample preparation for viral NGS (Fig. 1). Overall, large contigs matched to one of these eight viral families (Astroviridae, Caliciviridae, Nodaviridae, Leviviridae, Microviridae, Picornaviridae, Picobirnaviridae and Reoviridae) (Fig. 2). These families were detected in multiple samples and we focused on these families for the remaining analyses. No sequence with homology to the Hepatovirus genus or Hepeviridae family were detected.

\section{Norovirus Concentrations in Sewage Samples}

Quantification performed on four samples displayed comparable concentration (around $10^{3}$ RNA copies (c)/uL) for norovirus (Table 1). Norovirus GI was detected in all samples, with concentrations 10 to 100 times lower compare to norovirus GII concentrations. After PGM capture (method C) norovirus concentrations was similar for three samples, but was lower for sample 1920, both for genogroup I and II (Table 1).

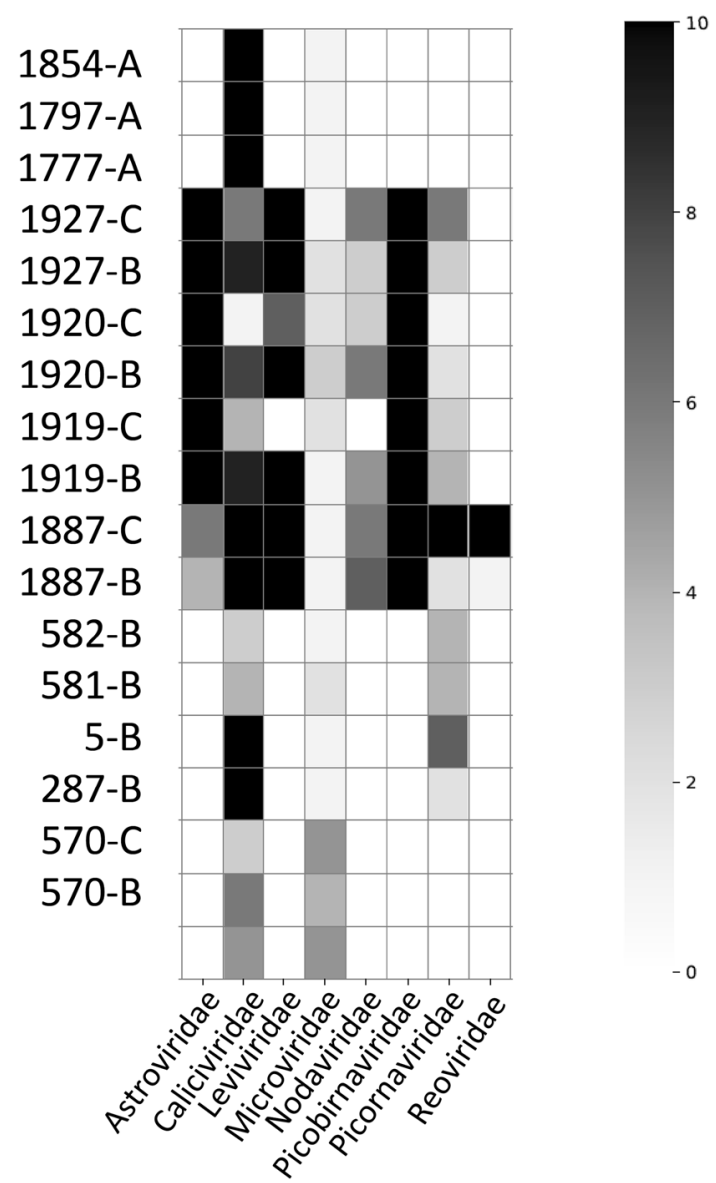

Fig. 2 Heat map of larger viral contig yield. Quality-controlled shortread data were de novo assembled into large contigs and contigs were classified at the protein level by comparison with virus familyspecific reference sets (see Methods). The numbers of contigs in each sample with $>60 \%$ protein identity and $500 \mathrm{nt}$ minimum contig length were plotted in a heat map. The colorbar to the right indicates number of contigs detected per sample in each virus family

\section{Utility of Target Enrichment (Method A) to Study Norovirus Diversity}

Targeting norovirus sequences using the norovirus enrichment capture allowed the recovery of long fragments with good coverage as the lowest count was 8.225 (Table 2). Almost complete genomes were obtained for seven GII strains and for two GI strains. Half of a GIV.1 norovirus sequence was also obtained. Another advantage of this approach is the sensitivity as the full-genome sequence for GI strain was obtained from sewage samples 1777 and 1854, having both a quite low concentration of $3 \mathrm{RNAc} / \mu \mathrm{L}$. 
Table 1 Norovirus concentrations in wastewater samples used for this study and corresponding calicivirus reads

\begin{tabular}{llrrr}
\hline Sample & Method & \multicolumn{2}{c}{ Norovirus* $^{2}$} & \multicolumn{1}{c}{ Calicivirus } \\
\cline { 3 - 4 } & & GI & GII & reads \\
\hline E1777 & A & 3 & 497 & NA \\
E1797 & A & 64 & 39 & NA \\
E1854 & A & 3 & 37 & NA \\
E1887 & B & 7 & 219 & 2507 \\
& C & 2 & 223 & 13,548 \\
E1919 & B & 19 & 361 & 3242 \\
& C & 6 & 346 & 10,635 \\
E1920 & B & 20 & 466 & 10,406 \\
& C & 3 & 81 & 3962 \\
E1927 & B & 26 & 140 & 1750 \\
& C & 11 & 637 & 2777 \\
\hline
\end{tabular}

NA data not collected

*Concentrations obtained using digital RT-PCR are expressed as RNA copies/ $\mu \mathrm{L}$ for genogroup I (GI) and II (GII)

**Number of reads mapped on contigs $>500$ nt using Bowtie 2

Table 2 Norovirus strains identified in sewage samples using method A

\begin{tabular}{llll}
\hline Sample & Contig length & Genotype* & Coverage** $^{* *}$ \\
\hline E1777 & 7278 & GIIP21-GII.3 & 19.586 \\
& 7484 & GIIP7-GII.6 & 99.576 \\
& 6828 & GIIPe-GII.4syd-v2 & 23.144 \\
& 7497 & GIIPe-GII4syd & 21.732 \\
& 7648 & GIP3-GI.3 & 16.817 \\
E1797 & 7343 & GIIP17-GII.7 & 85.503 \\
& 7431 & GIIP7-GII.6 & 47.253 \\
& 3467 & GIP9-GI.9 & 12.576 \\
& 7679 & GIPb-GI.6 & 71.396 \\
E1854 & 1582 & GIIP16-GII.13 & 12.72 \\
& 6509 & GIIP17-GII.17 & 8.225 \\
& 1221 & GIIPg & 8.663 \\
& 7641 & GIPb-GI.6 & 15.532 \\
& 3687 & GIV & 12.327 \\
\hline
\end{tabular}

*Sequences were identified using the online norovirus genotyping tool v2.0 (Kroneman et al. 2011)

**Coverage was calculated using Bowtie2 (Langmead et al. 2012)

\section{Impact of PEG Precipitation (Method B) on Virus Sequence Yield}

Viruses were first concentrated using high-molecular weight PEG precipitation for both stool and sewage samples. PEG has been used to concentrate enteric virus in oysters and water samples (Lewis et al. 1988) and when applied here, it was efficient in recovering long norovirus contigs, with
Table 3 Norovirus and other human virus detected in stool samples using method B

\begin{tabular}{llcl}
\hline Sample & Virus & Contig length & Genotype \\
\hline S570 & Norovirus & 7609 & GIIPe-GII.4 syd \\
S287 & Norovirus & 7542 & GIIP21-GII.3 \\
& Norovirus & 1104 & GIIP17-GII.17 \\
& Enterovirus & 653 & coxsackievirus A9 \\
S5 & Norovirus & 7390 & GIIP4NewOrleans-GII.4syd \\
& Norovirus & 3399 & GII.P21-GII.3 \\
& Enterovirus & 1387 & coxsackievirus A9 \\
S581 & Norovirus & 7528 & GIIP17-GII.17 \\
& Enterovirus & 1421 & coxsackievirus A9 \\
S582 & Norovirus & 7536 & GIIP17-GII.17 \\
& Enterovirus & 1363 & coxsackievirus A9 \\
\hline
\end{tabular}

five complete norovirus genomes obtained. An additional advantage of the PEG approach when combined with random primed deep sequencing was the detection of other enteric viruses present in the samples such as coxsackievirus in stool samples (Table 3), sapovirus, astrovirus and rotavirus in sewage samples (Table 4).

\section{Impact of Mucin Capture (Method C) on Virus Sequence Yield}

We aimed to investigate if a mucin capture step could provide enrichment of norovirus and other enteric virus materials. Calicivirus read numbers increased after mucin capture except for sample1920 for which norovirus GI and GII concentrations were also lower suggesting a failure in one purification step or a selection of some norovirus strains by the PGM (Table 1). No reproductible impact of the mucin capture on contig lengths was observed. Examining the lengths of norovirus contigs as a measure of success, four norovirus contigs were longer with mucine capture, while six norovirus contigs showed reduced lengh with mucin capture (compared to contig yield without PGM capture, Method B) (Table 4). An unexpected observation was the impact of this treatment on a number of other viruses as shown by the viral family-specific read yields for eight virus families. For Astroviridae, Reoviridae, Nodaviridae and Picobirnaviridae families, the yield of specific reads (as a percentage of total reads for that sample) was increased, as reported in upper panels of Fig. 3 comparing orange markers (method B) to blue markers (method C). This was confirmed by the identification of a complete genome for a human rotavirus A genotype G9-[P8], and for astrovirus (full genome for one strain and four additional strains identified) compared to method B (Table 4). In contrast, the yields of Leviviridae and Picornaviridae families remained similar or slightly reduced. A large amount of the phage PhiX174 (a member 
Table 4 Human enteric viruses detected using method $\mathrm{B}$ and $\mathrm{C}$

\begin{tabular}{|c|c|c|c|c|c|c|}
\hline \multirow[t]{2}{*}{ Sample } & \multicolumn{3}{|l|}{ Method B } & \multicolumn{3}{|l|}{ Method C } \\
\hline & Virus & Contig & Genotype & Virus & Contig & Genotype* \\
\hline S570 & Norovirus & 7609 & GIIPe-GII.4 & Norovirus & 6976 & GIIPe-GII.4 \\
\hline \multirow[t]{8}{*}{ E1887 } & Sapovirus & 7414 & GI.2 & Sapovirus & 7491 & GI.2 \\
\hline & Norovirus & 3241 & GIIP22-GII.17 & Norovirus & 2366 & GII.P22 \\
\hline & Norovirus & 2961 & GII & Norovirus & 2129 & GII \\
\hline & Norovirus & 2996 & GII.P7-GII.17 & Norovirus & 3126 & GII.P17-GII.17 \\
\hline & & & & Norovirus & 3024 & GII.P17-GII.6 \\
\hline & Rotavirus & 836 & & Rotavirus & 3283 & G9[P8] \\
\hline & Astrovirus & 3860 & Type 2 & Astrovirus & 6519 & Type 2 \\
\hline & & & & Husavirus & 494 & Sp.16915_89 \\
\hline \multirow[t]{4}{*}{ E1919 } & Norovirus & 4598 & GII.P16-GII.2 & Norovirus & 1028 & GIIP16 \\
\hline & Norovirus & 2692 & GII & Norovirus & 3004 & GII.P16-GII.4 syd \\
\hline & Sapovirus & 1209 & GII.3 & & & \\
\hline & Astrovirus & 6810 & Type 1 & Astrovirus & 6412 & Type 1 \\
\hline \multirow[t]{3}{*}{ E1920 } & Norovirus & 2239 & GII & Norovirus & 510 & GII.2 \\
\hline & Norovirus & 332 & GI-GI.3 & & & \\
\hline & Astrovirus & 6782 & Type 1 & Astrovirus & 1878 & Type 1 \\
\hline \multirow[t]{7}{*}{ E1927 } & Norovirus & 944 & GII.2 & Norovirus & 631 & GII.Postdam \\
\hline & & & & Norovirus & 616 & GII \\
\hline & Norovirus & 442 & GI & Norovirus & 631 & GI-GI.3 \\
\hline & & & & Sapovirus & 571 & GI.1 Seoul \\
\hline & Astrovirus & 1878 & Type 1 & Astrovirus & 6588 & Type 5 \\
\hline & & & & Astrovirus & 3948 & Type 1 \\
\hline & & & & Aichi virus & 2692 & Type 1 \\
\hline
\end{tabular}

*Strain identification was assigned using the online Norovirus genotyping tool v2.0 (Kroneman et al. 2011) of the Microviridae family) was added as a carrier after the PGM capture, thus we cannot make conclusion about the impact of PGM on the Microviridae family.

\section{Discussion}

This work aimed to generate enteric virus sequence data from human stool and sewage samples, with a special interest in norovirus. There are several challenges when applying metagenomics deep sequencing to describe the total virome of sewage samples (Bibby et al. 2013, Adriaenssens et al. 2018). One challenge is the high levels of dilution of human enteric viruses and the presence of a large variety of other microorganisms, plant, algae, chemical and organic compounds. Indeed, in non-outbreak settings, human enteric viruses are present at lower concentrations compared to bacteria or phages. In a previous study, we demonstrated that at least $1-4 \%$ of the population need to be infected to detect hepatitis $E$ virus in raw sewage from a small sewage treatment plant, showing the potential to rapidly detect outbreak in the population (Miura et al. 2016). Another challenge of sewage virome characterization is to obtain a representative sample of the viral load and diversity from the wastewater. Concentration of large volumes of water are currently used to decrease the sampling variability and increase the sensitivity threshold of the assays (Lewis et al. 1988; Schaeffer et al. 2018; Fernandez-Cassi et al. 2018). However, this may lead to increase concentration of inhibitors of the enzymes used for molecular assays, as human sewage may contain detergents, medicine, food additives, food waste and other chemicals and thus some purification steps are needed (Hata et al. 2017). Composite samples obtained over $24 \mathrm{~h}$, as used for this study, may be a convenient way to overcome some of these problems as it can be representative of what is entering in the sewage treatment plant without the need of large volumes. Different methods previously developed for sewage, water or shellfish analysis use the PEG precipitation that help to concentrate biomolecules by altering their hydration from solution and viruses (Lee et al. 1981; Lewis et al. 1988; Metcalf et al. 1995; Jiang et al.1992). This easy-to-use method was proposed for the first assay able to detect norovirus in stool (Jiang et al. 1992) and in preliminary test we verified that it helped to recover the complete norovirus genome from stool samples (data not shown). As human enteric viruses tend to aggregate or to bind to different types of particles, 

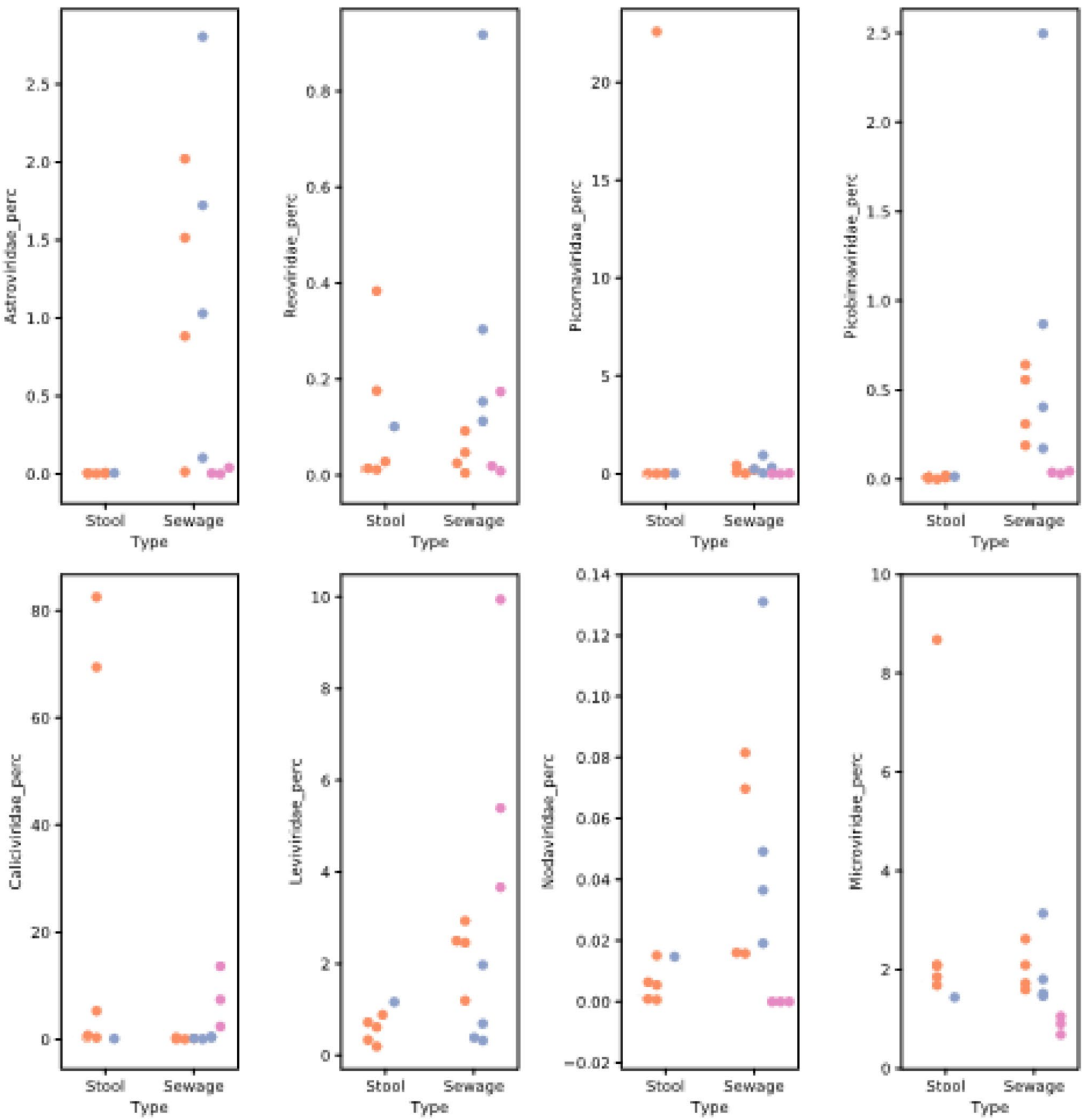

Fig. 3 Scatter plot of reads identified for each of 8 virus families (percentage of total reads). Quality-controlled reads for each of the 18 sample/conditions were mapped to virus family reference sets (see Methods) for Astroviridae, Caliciviridae, Nodaviridae, Leviviridae, Microviridae, Picornaviridae, Picobirnaviridae and Reoviridae. The

including bacteria, we applied an elution step based on $\mathrm{pH}$ variations under controlled conductivity conditions (Miura et al. 2013; Samandoulgou et al. 2015; Mullendore et al. 2001; da Silva et al. 2011). This added step may explain the higher efficacy to obtain long sequence of

number of reads identified in each family was plotted as percentage of the total quality-controlled reads for that read set. Data sets were separated into sample types (stool or sewage) and colored according sample treatment (method A: pink, method B: orange and method C: blue)

human enteric viruses compared to a published study that failed to identified norovirus strains (Hjelmso et al. 2017).

Capsid structures of a number of human enteric viruses including norovirus and rotavirus have been found to interact with the mucin family of glycoproteins due to the presence 
of binding sites for the complex carbohydrates on mucin (Le Pendu et al. 2014). This binding affinity was proposed to select norovirus particles from water samples using human histo-blood group antigen or PGM that present similar structure as it may increase the specificity by capturing non-damaged capsid and the sensitivity of detection by facilitating inhibitor removal (Zhou et al. 2017; Tian et al. 2008). In this study, PGM capture increased norovirus concentrations in the nucleic acid extracts, however, no difference was observed in terms of contig lengths or diversity of identified sequences. Combining PGM and HBGA type-B antigen could have help to improve this approach (Tian et al., 2017). One beneficial impact of the PGM capture was the detection of full genome of a rotavirus genogroup A genotype G9-[P8], confirming the binding affinity of rotavirus strains to glycans (Hu et al. 2018). When compared to method B (PEG without mucin capture), rotavirus was identified but full genome was not obtained, suggesting that PGM combined with random primed deep sequencing would be superior to detect full genomes of norovirus and rotavirus. It was more surprising to find a beneficial impact of PGM capture on astrovirus, with an increased diversity of strains identified. The PGM capture led also to the characterization of a few sequence of husavirus strain. Some husaviruses have been described as a novel virus family within the order Picornavirales, and are common in human fecal material (Oude Munnink et al. 2015). However, very little is known about astrovirus or husavirus PGM interactions, which may be due to non-specific interactions such as sialic acid recognition or other glycan affinity to viral capsid structure (Shanker et al. 2017; Hu et al. 2018). Adding purification steps enhance the risk to lose viruses as observed for one sample and also to introduce some bias of selection. Furthermore, when dealing with complex samples with a mixture of low amounts of viral contaminantions such as sewage, this may be an issue which need to be further investigated. Three biological replicates were found to be an useful approach to minimize potential biases and to give more confidence in analyzing aquatic viromes (Kim et al. 2017).

One objective of this work was to evaluate norovirus diversity present in stool and sewage samples. Some works based on a metabarcoding approach targeting the ORF2 portion of norovirus genome were successfully applied to sewage samples, but this approach limits the identification of strains that are not amplified by published primers (Oshiki et al. 2018; Fumian et al. 2019). In our work, we aimed to identify long fragments to be able to capture the diversity of strains circulating in the local population. The SureSelect target enrichment (method A) gave the highest number of full genome for several norovirus strains despite similar norovirus concentrations when compared with sewage samples used (Brown et al. 2016). This method was the only one able to yield norovirus GI strain identifications, important criteria for environmental sample analysis considering the importance of these strains transmission through the environment (Le Guyader et al. 2012; Verhoef et al. 2015). Although the SureSelect target enrichment array is designed to capture Caliciviridae sequences, these targeted sequences were still less than $1 \%$ of the total reads after capture, suggesting non-specific binding. Since the binding and wash conditions are propriety to the manufacturer and the actual bait concentrations are not provided, specificity improvements are limited. This method also allowed to identify a norovirus GIV.1 strain. This genogroup is sporadically detected in sewage samples, and may be under-appreciated as a cause of gastroenteritis, presumably because only a small number of sequences are available in GenBank causing difficulties in primer design (Sima et al. 2011; La Rosa et al. 2012). The two other methods also allowed to characterize some norovirus sequence, all of them being already reported in the NoroNet network (van Beek et al. 2018). Beside the ubiquitous GII.4 strains that have been reported worldwide, it was interesting to detect the GII.P17-GII.17 strain that caused several gastroenteritis outbreaks in multiple countries during this sampling period (Matsushima et al. 2015; Koo et al. 2017). In this regard, a complete characterization of detected viruses is important to identify new strains and thus to help risk manager to take measure to prevent further distribution (Cocolin et al. 2018).

A limitation of this study lies in the small sample size tested. Ideally, it would be more informative to have a larger number of samples being tested for the same approach to evaluate the method efficiency across sample variability. Sequence contents of raw sewage reflect the composition of the microbiome of local population and the diversity of all pathogenic or non-pathogenic bacteria or viruses circulating in the community at a larger extent than individual samples (Newton et al. 2015; Sano et al. 2016). To access this sequence information, we need to develop reproducible, simple, fast and easy to apply methods. The viral agnostic metagenomics approach is still an expensive approach when considering the library preparation or sequencing runs, but all the steps of the methods described here can easily be performed in environmental laboratory already performing PCR analysis as it just needs a centrifuge and basic equipment.

Acknowledgements We acknowledge the help of the SAUR and SUEZ technicians for sampling. We are most grateful to the Genomics and Bioinformatics Core Facility of Nantes (GenoBird, Biogenouest) for the technical support.

Funding This work was funded by EU-H2020 Grant No. 643476 COMPARE. My V.T. Phan is supported by Marie Sklodowska-Curie Individual Fellowship, funded by European Union's Horizon 2020 research and innovation programme (Grant Agreement No. 799417).

Data Availability The short-reads data for this study has been deposited in the European Nucleotide Archive (ENA) http://www.ebi.ac.uk/ena/ 
data/view/PRJEB31600 and to Genbank with the following (temporary) accession number: from MK789654 to MK789656 for NoV GI, from MK907785 to MK907802 for NoV GII, MK726262 for NoV GIV.

Open Access This article is distributed under the terms of the Creative Commons Attribution 4.0 International License (http://creativeco mmons.org/licenses/by/4.0/), which permits unrestricted use, distribution, and reproduction in any medium, provided you give appropriate credit to the original author(s) and the source, provide a link to the Creative Commons license, and indicate if changes were made.

\section{References}

Adriaenssens, E. M., Farkas, K., Harrison, C. H., Jones, D. L., Allison, H. E., \& McCarthy, A. J. (2018). Viromic analysis of wastewater input to a river catchment reveals a diverse assemblage of RNA viruses. mSystems, 3, e00015-00018.

Alberti, A., et al. (2017). Viral to metazoan marine plankton nucleotide sequences from the Tara Oceans expedition. Scientific Data, 4, 170093.

Atmar, R. L., Ramani, S., \& Estes, M. K. (2018). Human noroviruses: Recent advances in a 50-year history. Current Opinion in Infectious Disease, 31, 422-432.

Bankevich, A., Nurk, S., Antipov, D., Gurevich, A. A., et al. (2012). SPAdes: A new genome assembly algorithm and its applications to single-cell sequencing. Journal of Computational Biology, 19, 455-477.

Bibby, K., \& Peccia, J. (2013). Identification of viral pathogen diversity in sewage sludge by metagenome analysis. Environmental Science and Technology, 47, 1945-1951.

Brown, J. R., Roy, S., Ruis, C., Romero, E. Y., Shah, D., Willimas, R., et al. (2016). Norovirus whole-genome sequencing by sure select target enrichment: A robust and sensitive method. Journal of Virology, 54, 2530-2537.

Cocolin, L., Mataragas, M., Bourdichon, F., Doulgeraki, A., Pilet, M.-F., Jagadeesan, B., et al. (2018). Next generation microbiological risk assessment meta-omics: The next need for integration. International Journal of Food Microbiology, 287, 10-17.

Cotten, M., \& Koopmans, M. (2016). Next-generation sequencing and norovirus. Future Virology, 11, 719-722.

Cotten, M., Munnink, B. O., Canuti, M., Deijs, M., Watson, S. J., Kellam, P., et al. (2014). Full genome virus detection in fecal samples using sensitive nucleic acid preparartion, deep sequencing, and a novel iterative sequence classification algorithm. PLOS ONE, 9, e93269.

da Silva, A., Kavanagh, O. V., Estes, M. K., \& Elimelech, M. (2011). Adsorption and aggregation properties of norovirus GI and GII virus like particles demonstrate differeing responses to solution chemistry. Environmental Science and Technology, 45, 520-526.

de Graaf, M., Villabruna, N., \& Koopmans, M. P. G. (2017). Capturing norovirus transmission. Current Opinion in Virology, 22, 64-70.

Edgar, R. C. (2010). Search and clustering orders of magnitude faster than BLAST. Bioinformatics, 26, 2460-2461.

Fernandez-Cassi, X., Timoneda, N., Partinez-Puchol, S., Rusinol, M., Rodriguez-Manzano, J., Figuerola, N., et al. (2018). Metagenomics for the study of viruses in urban sewage as a tool for public health surveillance. Science of the Total Environment, 618, 870-880.

Forbes, J. D., Knox, N. C., Ronholm, J., Pagotto, F., \& Reimer, A. (2017). Metagenomics: The next culture-independant game changer. Frontiers in Microbiology, 8, 1069.
Fumian, T. M., Fioretti, J. M., Lun, J. H., dos Santos, I. A. L., White, P. A., \& Miagostovich, M. P. (2019). Detection of norovirus epidemic genotypes in raw sewage using next generation sequencing. Environment International, 123, 282-291.

Gerba, C., Betancourt, W. Q., Kitajima, M., \& Rock, C. M. (2018). Reducing uncertainty in estimating virus reduction by advanced water treatment process. Water Research, 133, 282-288.

Hata, A., Inaba, M., Katayama, H., \& Furumai, H. (2017). Characterization of natural organic substances potentially hindering RT-PCR-based virus detection in large volumes of environmental water. Environmental Science and Technology, 51, 13568-13579.

Hjelmso, M. H., Hellmer, M., Fernandez-Cassi, X., Timoneda, N., Lukjancenko, O., Seidel, M., et al. (2017). Evaluation of methods for the concentration and extraction of viruses from sewage in the context of metagenomic sequencing. PLOS ONE, 12, e0170199.

Hoque, S. A., Thongprachum, A., Takanashi, S., Mostafa, S. M., Saito, H., Anwar, K. S., et al. (2019). Alarming situation of spreading enteric viruses through sewage water in Dhaka city: Molecular epidemiological evidences. Food and Environmental Virology, $11,65-75$.

Hu, L., Sankaran, B., Laucirica, D. R., Estes, M. K., \& Prasad, B. V. V. (2018). Glycan recognition in globally dominant human rotaviruses. Nature Communications, 9, 2631.

Jiang, X., Wang, J., Graham, D. Y., \& Estes, M. K. (1992). Detection of Norwalk virus in stool by polymerase chain reaction. Journal of Clinical Microbiology, 30, 2529-2534.

Kim, Y., van Bonn, W., Aw, T. G., \& Rose, J. B. (2017). Aquarium viromes: Viromes of human-managed aquatic systems. Frontiers in Microbiology, 8, 1231.

Koo, E. S., Kim, M. S., Choi, Y. S., Park, K.-S., \& Jeong, Y. S. (2017). Occurrence of novel GII.17 and GII.21 norovirus variants in the coastal environment of South Korea in 2015. PLOS ONE, 12, e0172237.

Kroneman, A., Vennema, H., Deforche, K., Avoort, H.v.d., Peñaranda, S., Oberste, M.S., et al. (2011). An automated genotyping tool for enteroviruses and noroviruses. Journal of Clinical Virology, $51,121-125$.

La Rosa, G., Fratini, M., Vennarucci, V. S., Guercio, A., Purpari, G., \& Muscillo, M. (2012). GIV noroviruses and other enteric viruses in bivalves: A preliminary study. New Microbiologica, 35, 27-34.

Langmead, B., \& Salzberg, S. (2012). Fast gapped-read aligment with Bowtie 2. Nature Methods, 9, 357-359.

Le Guyader, F. S., Atmar, R. L., \& Le Pendu, J. (2012). Transmission of viruses through shellfish: When specific ligands come into play. Current Opinion in Virology, 2, 103-110.

Le Pendu, J., Nystrom, K., \& Ruvoen-Clouet, N. (2014). Host pathogen co-evolution and glycan interactions. Current Opinion in Virology, 7, 88-94.

Lee, J. C., \& Lee, L. L. Y. (1981). Preferential solvent interactions between proteins and polyethylene glycol. Journal of Biology and Chemistry, 256, 625-631.

Lewis, G. D., \& Metcalf, T. G. (1988). Polyethylene glycol precipitation for recovery of pathogenic virues, including Hepatitis A virus and human rotavirus, from oyster, water and sediment samples. Applied and Environmental Microbiology, 54, 1983-1988.

Matsushima, Y., Ishikawa, M., Shimizu, T., Komane, A., Kasuo, S., Shinohara, M., et al. (2015). Genetic analyses of GII.17 norovirus strains in diarrheal disease outbreaks from December 2014 to March 2015 in Japan reveal a novel polymerase sequence and amino acid substitution in the capsid region. Euro Surveillance, 20, 21173.

Metcalf, T., Melnick, J. L., \& Estes, M. K. (1995). Environmental Microbiology: From detection of virus in sewage and water by isolation to identification by molecular biology-A trip of over 50 years. Annual Review in Microbiology, 49, 461-487. 
Miura, T., Lhomme, S., Le Saux, J.-C., Le Mehaute, Ph, Guillois, Y., Couturier, E., et al. (2016). Detection of hepatitis E virus in sewage after an outbreak on a French island. Food and Environmental Virology, 8, 194-199.

Miura, T., Sano, D., Suenaga, A., Yoshimura, T., Fusawa, M., Nakagomi, T., et al. (2013). Histo-blood group antigen-like substances of human enteric bacteria as specific adsorbents for human noroviruses. Journal of Virology, 87, 9441-9451.

Mullendore, J., Sobsey, M. D., \& Shieh, Y. S. C. (2001). Improved method for the recovery of hepatitis A virus from oysters. Journal of Virological Methods, 94, 25-35.

Newton, R., McLellan, S. L., Dila, D. K., Vineis, J. H., Morrison, H. G., Eren, A. M., et al. (2015). Sewage reflects the microbiomes of human populations. mBio, 6, e02574-02584.

Nieuwenhuijse, D., \& Koopmans, M. P. G. (2017). Metagenomic sequencing for surveillance of food- and waterborne viral diseases. Frontiers in Microbiology, 8, 230.

Oakeson, K. F., Wagner, J. M., Mendenhall, M., Rohrwasser, A., \& Atkinson-Dunn, R. (2017). Bioinformatic analyses of wholegenome sequence data in a Public Health Laboratory. Emerging and Infectious Disease, 23, 1441-1445.

Oshiki, M., Miura, T., Kazama, S., Segawa, T., Ishii, S., Hatamoto, M., et al. (2018). Microfluidic PCR amplification and MiSeq amplicon sequencing techniques for high-throughput detection and genotyping of human pathogenic RNA viruses in human feces, sewage, and oysters. Frontiers in Microbiology, 9, 830.

Oude Munnink, B. B., Cotten, M., Deijs, M., Jebbink, M. F., Bakker, M., Mohammad, S., et al. (2015). A novel genus in the order Picornavirales detected in human stool. Journal of General Virology, 96, 3440-3443.

Polo, D., Schaeffer, J., Fournet, N., Le Saux, J.-C., Parnaudeau, S., McLeod, C., et al. (2016). Digital PCR for quantifying norovirus in oysters implicated in outbreaks, France. Emerging and Infectious Disease, 22, 2189-2191.

Samandoulgou, I., Fliss, I., \& Jean, J. (2015). Zeta potential and aggregation of virus-like partcile of human norovirus and feline calicivirus under different physicochemical conditions. Food and Environmental Virology, 7, 249-260.

Sano, D., Amarasiri, M., Hata, A., Watanabe, T., \& Katayama, H. (2016). Risk managment of viral infectious diseases in watewater reclamation. Environment International, 91, 220-229.

Schaeffer, J., Treguier, C., Piquet, J.-C., Cochennec-Laureau, N., Le Saux, J.-C., Garry, P., et al. (2018). Improving the efficacy of sewage tratment decrease norovirus contamination in oysters. International Journal of Food Microbiology, 286, 1-5.
Shanker, S., Hu, L., Ramani, S., Atmar, R. L., Estes, M. K., \& Prasad, B. V. V. (2017). Structural features of glycan recognition among viral pathogens. Current Opinion in Structural Biology, 44, 211-218.

Sima, L. C., Schaeffer, J., Le Saux, J.-C., Parnaudeau, S., Elimelech, M., \& Le Guyader, F. S. (2011). Calicivirus removal in a membrane bioreactor wastewater treatment plant. Applied and Environmental Microbiology, 77, 5170-5177.

Smith, D. B., Paddy, J. O., \& Simmonds, P. (2016). The use of human sewage screening for community surveillance of hepatitis $\mathrm{E}$ virus in the UK. Journal of Medical Virology, 88, 915-918.

Tian, P., Engelbrektson, A., \& Mandrell, R. E. (2008). Two-log incrase in sensitivity for detection of norovirus in complex samples by concentration with porcine gastric mucin conjugated to magnetic beads. Applied and Environmental Microbiology, 74, 4271-4276.

Tian, P., Yang, D., Shan, L., Li, Q., Liu, D., \& Wang, D. (2017). Estimation of Human norovirus infectivity from environmental water samples by in situ capture RT-qPCR method. Food and Environmental Virology, 10, 29-38.

van Beek, J., de Graaf, M., Al-Hello, H., Ambert-Balay, K., Botteldoorn, N., Brytting, M., et al. (2018). Molecular surveillance of norovirus, 2005-2016: An epidemiological analysis of data collected from the NoroNet network. Lancet Infectious Disease, 18, $545-553$.

Verhoef, L., Hewitt, J., Barclay, L., Ahmed, S. M., Lake, R., Hall, A. J., et al. (2015). Norovirus genotype profiles associated with foodborne transmission, 1999-2012. Emerging and Infectious Disease, 21, 592-599.

Watson, S. J., Welkers, M. R. A., Depledge, D. P., Coulter, E., Breuer, J. M., de Jong, M. D., et al. (2013). Viral population analysis and minority-variant detection using short read next-generation sequencing. Philosophical Transactions of the Royal Society B, 368, 20120205.

Zhou, Z., Tian, Z., Li, Q., Tian, P., Wu, Q., Wang, D., et al. (2017). In situ capture RT-qPCR: A new simple and sensitive method to detect human norovirus in oysters. Frontiers in Microbiology, 8 , 554.

Publisher's Note Springer Nature remains neutral with regard to jurisdictional claims in published maps and institutional affiliations. 